VOL. 52 (1995) [215-224]

\title{
UNICITY THEOREMS FOR MEROMORPHIC OR ENTIRE FUNCTIONS II
}

\author{
HONG-XUN YI
}

In 1976, Gross posed the question "can one find two (or possibly even one) finite sets $S_{j}(j=1,2)$ such that any two entire functions $f$ and $g$ satisfying $E_{f}\left(S_{j}\right)=$ $E_{g}\left(S_{j}\right)$ for $j=1,2$ must be identical?", where $E_{f}\left(S_{j}\right)$ stands for the inverse image of $S_{j}$ under $f$. In this paper, we show that there exists a finite set $S$ with 11 elements such that for any two non-constant meromorphic functions $f$ and $g$ the conditions $E_{f}(S)=E_{g}(S)$ and $E_{f}(\{\infty\})=E_{g}(\{\infty\})$ imply $f \equiv g$. As a special case this also answers the question posed by Gross.

\section{INTRODUCTION}

By a meromorphic function we shall always mean a meromorphic function in the complex plane. It is assumed that the reader is familiar with the notation of Nevanlinna Theory (see, for example, [3]). We use $E$ to denote any set of positive real numbers of finite linear measure, not necessarily the same at each occurrence. For any nonconstant meromorphic function $f$, we denote by $S(r, f)$ any quantity satisfying

$$
S(r, f)=o(T(r, f)) \quad(r \rightarrow \infty, r \notin E) .
$$

For any set $S$ and any meromorphic function $f$ let

$$
E_{f}(S)=\bigcup_{a \in S}\{z \mid f(z)-a=0\}
$$

where each zero of $f-a$ with multiplicity $m$ is repeated $m$ times in $E_{f}(S)$.

In 1976, Gross proved [1] that there exist three finite sets $S_{j}(j=1,2,3)$ such that any two entire functions $f$ and $g$ satisfying $E_{f}\left(S_{j}\right)=E_{g}\left(S_{j}\right)$ for $j=1,2,3$ must be identical. In the same paper Gross posed the following question (see [1, Question 6]): Can one find two (or possibly even one) finite sets $S_{j}(j=1,2)$ such that any two non-constant entire functions $f$ and $g$ satisfying $E_{f}\left(S_{j}\right)=E_{g}\left(S_{j}\right)$ for $j=1,2$ must be identical?

The present author proved the following result which is partial answer of the above question.

Received 3 November 1994

Project supported by the National Natural Science Foundation of PRC.

Copyright Clearance Centre, Inc. Serial-fee code: 0004-9729/95 \$A2.00+0.00. 
Theorem A. (see [7, Theorem 3]). Let $S_{1}=\left\{w \mid w^{n}-1=0\right\}, S_{2}=\{a, b\}$, where $n(>6)$ is a positive integer, $a$ and $b$ are constants such that $a b \neq 0, a^{n} \neq b^{n}$, $a^{2 n} \neq 1, b^{2 n} \neq 1$ and $a^{n} b^{n} \neq 1$. Suppose that $f$ and $g$ are nonconstant entire functions satisfying $E_{f}\left(S_{j}\right)=E_{g}\left(S_{j}\right)$ for $j=1,2$. Then $f \equiv g$.

For the meromorphic case, the present author proved a corresponding theorem, which is the generalisation of Theorem A.

Theorem B. (see [7, Theorem 2]). Let $S_{1}$ and $S_{2}$ be defined as in Theorem $A$, and let $S_{3}=\{\infty\}$. Suppose that $f$ and $g$ are nonconstant meromorphic functions satisfying $E_{f}\left(S_{j}\right)=E_{g}\left(S_{j}\right)$ for $j=1,2,3$. Then $f \equiv g$.

The set $S$ such that for any two nonconstant entire functions $f$ and $g$ the condition $E_{f}(S)=E_{g}(S)$ implies $f \equiv g$ is called a unique range set (URS in brief) of entire functions (see [2]). In 1982, Gross and Yang proved the following result.

Theorem C. (see [2, Theorem 3]). The set $S=\left\{w \mid e^{w}+w=0\right\}$ is a URS of entire functions.

Note that the set $S=\left\{w \mid e^{w}+w=0\right\}$ contains an infinite number of elements and so Theorem $\mathrm{C}$ does not answer the question posed by Gross.

In this paper we give a positive answer to Gross' question. In fact, we prove more generally the following theorem.

TheOREM 1. Let $S=\left\{w \mid w^{n}+a w^{n-m}+b=0\right\}$, where $n$ and $m$ are two positive integers such that $n$ and $m$ have no common factors and $n \geqslant 2 m+5, a$ and $b$ are two nonzero constants such that the algebric equation $w^{n}+a w^{n-m}+b=0$ has no multiple roots. Then the set $S$ is a URS of entire functions.

By Theorem 1, we immediately obtain the following corollary.

Corollary 1. Let $S=\left\{w \mid w^{7}+a w^{6}+b=0\right\}$, where $a$ and $b$ are two non-zero constants such that $b \neq-6^{6}(a / 7)^{7}$. Then the set $S$ is a URS of entire functions with 7 elements.

For meromorphic functions, we have the following result, which is an extension of Theorem 1.

THEOREM 2. Let $S=\left\{w \mid w^{n}+a w^{n-m}+b=0\right\}$, where $n$ and $m$ are two positive integers such that $m \geqslant 2, n \geqslant 2 m+7$ with $n$ and $m$ having no common factors, $a$ and $b$ are two nonzero constants such that the algebric equation $w^{n}+a w^{n-m}+b=0$ has no multiple roots. Suppose that $f$ and $g$ are nonconstant meromorphic functions satisfying $E_{f}(S)=E_{g}(S)$ and $E_{f}(\{\infty\})=E_{g}(\{\infty\})$. Then $f \equiv g$.

By Theorem 2, we immediately obtain the following corollary.

COROLlary 2. Let $S=\left\{w \mid w^{11}+a w^{9}+b=0\right\}$, where $a$ and $b$ are two non-zero constants such that $b^{2} \neq-2^{2} 9^{9}(a / 11)^{11}$. Then for any two nonconstant meromorphic 
functions $f$ and $g$, the conditions $E_{f}(S)=E_{g}(S)$ and $E_{f}(\{\infty\})=E_{g}(\{\infty\})$ imply $f \equiv g$.

\section{SOME LEMMAS}

The following lemmas will be needed in the proof of our theorems.

Lemma 1. (see [6]). Let $f$ and $g$ be two nonconstant meromorphic functions, and let $c_{1}, c_{2}$ and $c_{3}$ be three nonzero constants. If

$$
c_{1} f+c_{2} g=c_{3}
$$

then

$$
T(r, f)<\bar{N}\left(r, \frac{1}{f}\right)+\bar{N}\left(r, \frac{1}{g}\right)+\bar{N}(r, f)+S(r, f)
$$

Lemma 2. (see [4]). Let $f_{1}, f_{2}, \ldots, f_{n}$ be linearly independent meromorphic functions satisfying

$$
\sum_{j=1}^{n} f_{j}=1
$$

Then for $k=1,2, \ldots, n$ we have

$$
\begin{aligned}
T\left(r, f_{k}\right)< & \sum_{j=1}^{n} N\left(r, \frac{1}{f_{j}}\right)+N\left(r, f_{k}\right)+N(r, D)-\sum_{j=1}^{n} N\left(r, f_{j}\right) \\
& -N\left(r, \frac{1}{D}\right)+o(T(r)) \quad(r \notin E),
\end{aligned}
$$

where $D$ denotes the Wronskian of the functions $f_{1}, f_{2}, \ldots, f_{n}$ and $T(r)$ denotes the maximum of $T\left(r, f_{j}\right), j=1,2, \ldots, n$.

LEMмa 3. (see [5]). Let $f$ be a nonconstant meromorphic function, and let $P(f)$ be a polynomial in $f$ of the form

$$
P(f)=a_{0} f^{n}+a_{1} f^{n-1}+\ldots+a_{n-1} f+a_{n}
$$

where $a_{0}(\neq 0), a_{1}, \ldots, a_{n}$ are constants. Then

$$
T(r, P(f))=n T(r, f)+S(r, f)
$$




\section{Proof of Theorem 2}

Let $w_{1}, w_{2}, \ldots, w_{n}$ be the roots of the equation $w^{n}+a w^{n-m}+b=0$. Since $E_{f}(S)=E_{g}(S)$ and $E_{f}(\{\infty\})=E_{g}(\{\infty\})$, we have from Nevanlinna's second fundamental theorem

$$
\begin{aligned}
(n-1) T(r, g) & <\sum_{j=1}^{n} N\left(r, \frac{1}{g-w_{j}}\right)+N(r, g)+S(r, g) \\
& =\sum_{j=1}^{n} N\left(r, \frac{1}{f-w_{j}}\right)+N(r, f)+S(r, g) \\
& \leqslant(n+1) T(r, f)+S(r, g) .
\end{aligned}
$$

Thus

$$
T(r, g)<\frac{n+1}{n-1} T(r, f)+S(r, g)
$$

In the same manner as above, we have

$$
T(r, f)<\frac{n+1}{n-1} T(r, g)+S(r, f)
$$

Again by $E_{f}(S)=E_{g}(S)$ and $E_{f}(\{\infty\})=E_{g}(\{\infty\})$, we obtain

$$
\frac{f^{n}+a f^{n-m}+b}{g^{n}+a g^{n-m}+b}=e^{h}
$$

where $h$ is an entire function. From Lemma $3,(1)$ and (3) we have

$$
\begin{aligned}
T\left(r, e^{h}\right) & \leqslant T\left(r, f^{n}+a f^{n-m}+b\right)+T\left(r, g^{n}+a g^{n-m}+b\right)+O(1) \\
& =n T(r, f)+n T(r, g)+S(r, f) \\
& <\frac{2 n^{2}}{n-1} T(r, f)+S(r, f) .
\end{aligned}
$$

Let us put

$$
\begin{aligned}
& f_{1}=-\frac{1}{b} f^{n-m}\left(f^{m}+a\right), \\
& f_{2}=e^{h}, \\
& f_{3}=\frac{1}{b} g^{n-m}\left(g^{m}+a\right) e^{h},
\end{aligned}
$$

and let $T(r)$ denote the maximum of $T\left(r, f_{j}\right), j=1,2,3$. From (3), (5), (6) and (7), we obtain

$$
f_{1}+f_{2}+f_{3}=1
$$


From (1), (4), (5), (6) and (7) we have

$$
T(r)=O(T(r, f)) \quad(r \notin E) .
$$

Next, we need the following lemma.

Lemma 4. $f_{1}, f_{2}$ and $f_{3}$ are linearly dependent.

Proof: Suppose that $f_{1}, f_{2}$ and $f_{3}$ are linearly independent. Applying Lemma 2 to the functions $f_{j}(j=1,2,3)$, from (8) and (9) we have

(10)

$$
\begin{aligned}
T\left(r, f_{1}\right)< & \sum_{j=1}^{3} N\left(r, \frac{1}{f_{j}}\right)-N\left(r, \frac{1}{D}\right)+N(r, D)-N\left(r, f_{2}\right)-N\left(r, f_{3}\right) \\
& +S(r, f)
\end{aligned}
$$

where

$$
D=\left|\begin{array}{lll}
f_{1} & f_{2} & f_{3} \\
f_{1}^{\prime} & f_{2}^{\prime} & f_{3}^{\prime} \\
f_{1}^{\prime \prime} & f_{2}^{\prime \prime} & f_{3}^{\prime \prime}
\end{array}\right| .
$$

From (5), (6) and (7) we have

$$
\begin{aligned}
\sum_{j=1}^{3} N\left(r, \frac{1}{f_{j}}\right)=( & n-m) N\left(r, \frac{1}{f}\right)+N\left(r, \frac{1}{f^{m}+a}\right) \\
& +(n-m) N\left(r, \frac{1}{g}\right)+N\left(r, \frac{1}{g^{m}+a}\right) .
\end{aligned}
$$

By looking at the zeros of $f$ and $g$, from (5), (6), (7) and (11) we see that

$$
N\left(r, \frac{1}{D}\right) \geqslant(n-m) N\left(r, \frac{1}{f}\right)-2 \bar{N}\left(r, \frac{1}{f}\right)+(n-m) N\left(r, \frac{1}{g}\right)-2 \bar{N}\left(r, \frac{1}{g}\right)
$$

From (8) and (11) we get

$$
D=\left|\begin{array}{ll}
f_{2}^{\prime} & f_{3}^{\prime} \\
f_{2}^{\prime \prime} & f_{3}^{\prime \prime}
\end{array}\right|
$$

Since $f_{2}$ is entire, from (7) and (14) we have

$$
\begin{aligned}
N(r, D)-N\left(r, f_{2}\right)-N\left(r, f_{3}\right) & \leqslant N\left(r, f_{3}^{\prime \prime}\right)-N\left(r, f_{3}\right) \\
& =2 \bar{N}\left(r, f_{3}\right)=2 \bar{N}(r, g)=2 \bar{N}(r, f) .
\end{aligned}
$$


From Lemma 3, (5), (10), (12), (13) and (15) we deduce

$$
\begin{aligned}
n T(r, f)< & 2 \bar{N}\left(r, \frac{1}{f}\right)+N\left(r, \frac{1}{f^{m}+a}\right)+2 \bar{N}\left(r, \frac{1}{g}\right) \\
& +N\left(r, \frac{1}{g^{m}+a}\right)+2 \bar{N}(r, f)+S(r, f) \\
& <(4+m) T(r, f)+(2+m) T(r, g)+S(r, f) .
\end{aligned}
$$

From this and (1) we obtain

$$
n T(r, f)<\left(2 m+6+\frac{2(2+m)}{n-1}\right) T(r, f)+S(r, f) .
$$

Since $n \geqslant 2 m+7,(16)$ is a contradiction, which proves Lemma 4 .

Next we proceed to prove Theorem 2.

By Lemma 4 we know that $f_{1}, f_{2}$ and $f_{3}$ are linearly dependent. Then there exist three constants $c_{1}, c_{2}$ and $c_{3}$, at least one of which is not zero, such that

$$
c_{1} f_{1}+c_{2} f_{2}+c_{3} f_{3}=0
$$

If $c_{1}=0$, from (17) we have $c_{2} \neq 0, c_{3} \neq 0$ and

$$
f_{3}=-\frac{c_{2}}{c_{3}} f_{2}
$$

Hence, from (6) and (7) we obtain

$$
g^{n}+a g^{n-m}=-b c_{2} / c_{3}
$$

which is impossible. Thus $c_{1} \neq 0$ and

$$
f_{1}=-\frac{c_{2}}{c_{1}} f_{2}-\frac{c_{3}}{c_{1}} f_{3}
$$

Now combining (8) and (18) we get

$$
\left(1-\frac{c_{2}}{c_{1}}\right) f_{2}+\left(1-\frac{c_{3}}{c_{1}}\right) f_{3}=1
$$

We discuss the following three cases. 
CASE I. Assume $c_{1} \neq c_{2}$ and $c_{1} \neq c_{3}$. From (6), (7) and (19) we have

$$
-\frac{1}{b}\left(1-\frac{c_{3}}{c_{1}}\right) g^{n-m}\left(g^{m}+a\right)+c^{-h}=1-\frac{c_{2}}{c_{1}}
$$

From (20) we know that $g$ is a entire function. By Lemma 1, Lemma 3 and (20) we obtain

$$
\begin{aligned}
n T(r, g) & <\bar{N}\left(r, \frac{1}{g^{n-m}\left(g^{m}+a\right)}\right)+S(r, g) \\
& =\bar{N}\left(r, \frac{1}{g}\right)+\bar{N}\left(r, \frac{1}{g^{m}+a}\right)+S(r, g) \\
& <(1+m) T(r, g)+S(r, g),
\end{aligned}
$$

which is impossible.

CASE II. Assume $c_{1}=c_{2}$. From (19) we have $c_{1} \neq c_{3}$ and

$$
f_{3}=\frac{c_{1}}{c_{1}-c_{3}}
$$

From (7) and (21) we get

$$
g^{n-m}\left(g^{m}+a\right)=\frac{b c_{1}}{c_{1}-c_{3}} e^{-h}
$$

Let $a_{1}, a_{2}, \ldots, a_{m}$ be the roots of equation $w^{m}+a=0$. From (22) we know that $\infty$, $0, a_{1}, a_{2}, \ldots, a_{m}$ are Picard exceptional values of $g$, which is impossible.

CASE III. Assume $c_{1}=c_{3}$. From (19) we have $c_{1} \neq c_{2}$ and

$$
f_{2}=\frac{c_{1}}{c_{1}-c_{2}}
$$

that is

$$
e^{h}=\frac{c_{1}}{c_{1}-c_{2}}
$$

From (5), (7), (8) and (23) we get

$$
-f^{n-m}\left(f^{m}+a\right)+\frac{c_{1}}{c_{1}-c_{2}} g^{n-m}\left(g^{m}+a\right)=\frac{b c_{2}}{c_{2}-c_{1}}
$$


If $c_{2} \neq 0$, by Lemma 1 and Lemma 3, we have from (24),

$$
\begin{aligned}
n T(r, f)< & \bar{N}\left(r, \frac{1}{f^{n-m}\left(f^{m}+a\right)}\right)+\bar{N}\left(r, \frac{1}{g^{n-m}\left(g^{m}+a\right)}\right)+\bar{N}(r, f)+S(r, f) \\
= & \bar{N}\left(r, \frac{1}{f}\right)+\bar{N}\left(r, \frac{1}{f^{m}+a}\right)+\bar{N}\left(r, \frac{1}{g}\right) \\
& \quad+\bar{N}\left(r, \frac{1}{g^{m}+a}\right)+\bar{N}(r, f)+S(r, f) \\
< & (2+m) T(r, f)+(1+m) T(r, g)+S(r, f) .
\end{aligned}
$$

From this and (1) we obtain

$$
n T(r, f)<\left(3+2 m+\frac{2(1+m)}{n-1}\right) T(r, f)+S(r, f),
$$

which is impossible. Thus $c_{2}=0$. From (24) we deduce

$$
f^{n}-g^{n}=-a\left(f^{n-m}-g^{n-m}\right) .
$$

If $f \not \equiv g$, from (25) we obtain

$$
g^{m}=-\frac{a(H-v)\left(H-v^{2}\right) \ldots\left(H-v^{n-m-1}\right)}{(H-u)\left(H-u^{2}\right) \ldots\left(H-u^{n-1}\right)}
$$

where $H=f / g, u=\exp ((2 \pi i) / n)$ and $v=\exp ((2 \pi i) /(n-m))$. From (26) we know that $H$ is a nonconstant meromorphic function. Since $n$ and $m$ have no common factors, we have $u^{j} \neq v^{k}(j=1,2, \ldots, n-1 ; k=1,2, \ldots, n-m-1)$. Suppose that $z_{j}$ is a zero of $H-u^{j}$ of order $p_{j}$. From (26) we have $p_{j} \geqslant m \geqslant 2$. Thus

$$
\bar{N}\left(r, \frac{1}{H-u^{j}}\right) \leqslant \frac{1}{m} N\left(r, \frac{1}{H-u^{j}}\right) \leqslant \frac{1}{2} T(r, H)+O(1) .
$$

By the second fundamental theorem, from (27) we obtain

$$
\begin{aligned}
(n-3) T(r, H) & <\sum_{j=1}^{n-1} \bar{N}\left(r, \frac{1}{H-u^{j}}\right)+S(r, H) \\
& <\frac{n-1}{2} T(r, H)+S(r, H),
\end{aligned}
$$

which is impossible. Hence, $f \equiv g$.

This completes the proof of Theorem 2 . 


\section{Supplement to Theorem 2}

It is reasonable to ask: What can be said if $m=1$ in Theorem 2 ? In this section, we prove the following theorem, which is a supplement to Theorem 2 .

Theorem 3. Let $S=\left\{w \mid w^{n}+a w^{n-1}+b=0\right\}$, where $n>8$, and $a$ and $b$ are two nonzero constants such that the algebraic equation $w^{n}+a w^{n-1}+b=0$ has no multiple roots. Suppose that $f$ and $g$ are two distinct nonconstant meromorphic functions satisfying $E_{f}(S)=E_{g}(S)$ and $E_{f}(\{\infty\})=E_{g}(\{\infty\})$. Then

$$
f=-\frac{a H\left(H^{n-1}-1\right)}{H^{n}-1} \quad \text { and } g=-\frac{a\left(H^{n-1}-1\right)}{H^{n}-1}
$$

where $H$ is a nonconstant meromorphic function.

Proof: Proceeding as in the proof of Theorem 2, we have

$$
f^{n}-g^{n}=-a\left(f^{n-1}-g^{n-1}\right) .
$$

Noting $f \not \equiv g$, from (28) we obtain

$$
g=-\frac{a\left(H^{n-1}-1\right)}{H^{n}-1}
$$

where $H=f / g$. From (29) we know that $H$ is a nonconstant meromorphic function. Thus, from (29) we have

$$
f=-\frac{a H\left(H^{n-1}-1\right)}{H^{n}-1}
$$

This completes the proof of Theorem 3.

\section{Proof OF ThEOREM 1}

If $f \not \equiv g$, noting $N(r, f)=N(r, g)=0$ and proceeding as in the proof of Theorem 2 , we can obtain (26). Since $g$ is a nonconstant entire function, from (26) we know that $u^{j}(j=1,2, \ldots, n-1)$ are Picard exceptional values of $H$, which is impossible. Thus $f \equiv g$, which proves Theorem 1 .

\section{References}

[1] F. Gross, 'Factorization of meromorphic functions and some open problems', in Complex Analysis, Lecture Notes in Math. 599, Proc. Conf. Univ. Kentucky, Lexington, Kentucky 1976 (Springer-Verlag, Berlin, Heidelberg, New York, 1977).

[2] F. Gross and C.C. Yang, 'On preimage range sets of meromorphic functions'; Proc. Japan Acad. 58 (1982), 17-20. 
[3] W.K. Hayman, Meromorphic functions (Clarendon Press, Oxford, 1964).

[4] R. Nevanlinna, Le théorème de Picard-Borel et la théorie des fonctions méromorphes (Gauthier-Villars, Paris, 1929).

[5] C.C. Yang, 'On deficiencies of differential polynomials II', Math. Z. 125 (1972), 107-112.

[6] Hong-Xun Yi, 'Meromorphic functions that share three values', Chinese Ann. Math. Ser. A 9 A (1988), 434-440.

[7] Hong-Xun Yi, 'Unicity theorems for meromorphic or entire functions', Bull. Austral. Math. Soc. 49 (1994), 257-265.

Department of Mathematics

Shandong University

Jinan

Shandong 250100

Peoples Republic of China 